\title{
Factors Affecting Mortality in Adult Tetanus Patients
}

\author{
Lohghinee Krisnan, ${ }^{1}$ Anam, ${ }^{2}$ Ramdan Panigoro ${ }^{3}$ \\ ${ }^{1}$ Faculty of Medicine Universitas Padjadjaran ${ }^{2}$ Department of Neurology Faculty of Medicine \\ Universitas Padjadjaran/Dr. Hasan Sadikin General Hospital Bandung, ${ }^{3}$ Department of \\ Biochemistry Faculty of Medicine Universitas Padjadjaran
}

\begin{abstract}
Background: Tetanus is a devastating disease that is associated with high mortality. The aim of this study was to analyze the prognostic factors that were associated with high mortality in tetanus.

Methods: This was an analytical study and the data was collected retrospectively from 1 January 2010-310ctober 2012 in the Department of Neurology, Dr. Hasan Sadikin General Hospital.

Results: Out of hundred thirty two cases only 87 cases of tetanus patient have complete medical records. There were 54 male and 33 female. The age range was 35-49 years old. Mortality rate was high $(32.18 \%)$. The most frequent wound site is extremities $(85.07 \%)$. The absence of post-injury tetanus vaccination was $75.86 \%$, period of onset $<48$ hours was $67.82 \%$ and the use of mechanical ventilation and tracheostomy $36.07 \%$. Mortality was significantly associated with an incubation period $<7$ days $(p<0.05)$, presence of fever $(p<0.05)$, co morbidity of autonomic storm $(p<0.01)$, and the severity of the disease by grade $5(p<0.05)$. Conclusions: Factors affecting the mortality in adult tetanus patients were significant for incubation period, fever, co morbidity and severity of the disease by grade. [AMJ.2015;2(1):157-61]
\end{abstract}

Keywords: Mortality, prognostic factors, tetanus

\section{Introduction}

Tetanus, described by Hippocrates as "a timeless human misery", still exists in the 21st century especially in the developing countries. Even with the invention of anti tetanus serum and tetanus toxoid by P. Descombey in 1924, as prevention tetanus immunization is not well practiced in most of the countries. Meanwhile, tetanus still remains as a public health problem in Indonesia. This disease is triggered by a microbiological organism called clostridium tetani which causes muscle spasm and lock jaw. Furthermore, in severe cases with increasing severity and frequency of spasms can lead to death.

In 1995, the World Health Organization (WHO) had an intention to eradicate tetanus however, in developing countriesitstill remains as endemic. ${ }^{1}$ In Indonesia, the surveillance data by the Ministry of Health showed the incidence of tetanus is about 0.2 per 100,000 populations annually. It is regarded as an even distribution of the disease in all provinces in the country. ${ }^{2}$ The incidence for the year 2011 in Indonesia is 210 , as stated by the WHO.
However, the incidence has shown a decline since $2003 .^{3}$

Tetanus is treated with intensive care, muscle relaxant drugs, and often assisted ventilation. Besides, anti-tetanus serum, tetanus toxoid and metronidazole are also administered. Proper wound debridement and management are also carried out. Active immunization of tetanus toxoid is given during childhood and continued with booster after 5 years to eradicate this disease. Furthermore, passive immunization is given following wound or injury. ${ }^{4}$ Despite the help of the current modern medical advances, the mortality for generalized tetanus is still high. Therefore, this research was to analyze the prognostic factors affecting the mortality in adult tetanus patients.

\section{Methods}

Adult tetanus patients admitted in the Department of Neurology in Dr. Hasan Sadikin General Hospital, Bandung, from 1 January 2010-310ctober 2012 were included in this analytical study. Data were collected

Correspondence: Lohghinee Krisnan, Faculty of Medicine, Universitas Padjadjaran, Jalan Raya Bandung-Sumedang Km.21, Jatinangor, Sumedang, Indonesia, Phone: +6283820120153 Email: viloni_24@yahoo.com 
retrospectively. Diagnosis of tetanus was based on Patel and Joag grading. Patients were divided into five grades based on the criteria such as incubation period, period of onset, fever, and spasm and lock jaw. Fever is defined as temperature $>37.5$. Grade I consisted of one of these criteria. For every additional criterion there will be alteration of the grade by one criterion. If all five criteria were present, it indicated grade $\mathrm{V}$. While mild is range from grade I-II, moderate range from III-IV and severe was grade $\mathrm{V}$.

The collected data included patient's details, age and sex, incubation period, period of onset, site of wound, immunization background, passive immunization, classification, grading, the comorbidity such as autonomic storm, cardiac arrest, aspiration pneumonia, and stress ulcer; and the use of mechanical ventilation. The incubation period was defined as a time period from time of injury to onset of symptoms, and the period of onset was defined as the time period from onset of symptoms to time of full blown disease / onset of general spasms. The patients also received passive immunization with anti tetanus serum 10,000 IU and active immunization with tetanus toxoid. Patients were graded based on the severity of the disease: mild, moderate and severe according to Patel and Joag.5 The data will be analyzed using Chi-squared tests or, when needed, the Fisher's exact test was used, to analyze the association between mortality and categorical variables. ${ }^{6}$

\section{Results}

Out of a total of 132 cases of tetanus only 87 patients with complete records were included in this study. Out of the 87 patients, 86 of them had generalized tetanus and only one of them had cephalic tetanus. All patients had spasms and lockjaw. There were $54(67.07 \%)$ male and $33(37.93 \%)$ female patients. The age range was between $20-80$ years. The highest number of patients presented with tetanus was between 35-49 years which was 34 (39.08\%).

Table 1 The Distribution of the Demographic data of Tetanus Patients

\begin{tabular}{lcc}
\hline \multicolumn{1}{c}{ Demographic data } & No. of patients & \% \\
\hline Sex & 54 & 67.07 \\
Male & 33 & 37.93 \\
Female & & \\
Age (years old) & 10 & 11.49 \\
$20-34$ & 34 & 39.08 \\
$35-49$ & 29 & 32.18 \\
$50-64$ & 14 & 16.09 \\
$>65$ & & \\
Wound site & 74 & 85.07 \\
Extremities & 11 & 12.65 \\
Face/nose/mouth & 2 & 2.30 \\
Trunk & & \\
Vaccine after injury & 21 & 24.14 \\
Yes & 66 & 75.86 \\
No & & \\
Grading* & 12 & 13.79 \\
2 & 45 & 51.72 \\
3 & 23 & 26.44 \\
4 & 7 & 8.05 \\
5 & $34-m 09$ & \\
\hline
\end{tabular}

Notes: ${ }^{*}$ Grading is according to Patel and Joag: 0-2=mild, 3-4=moderate, 5=severe 
Table 2 The Relationship between Mortality and Prognostic Factors for Tetanus

\begin{tabular}{|c|c|c|c|c|}
\hline Factors & Total $(n=87)$ & Died $(n=28)$ & Mortality rate $(\%)$ & P-value \\
\hline \multicolumn{5}{|l|}{ Sex } \\
\hline Male & 54 & 23 & 42.59 & \multirow[t]{2}{*}{0.189} \\
\hline Female & 33 & 5 & 15.15 & \\
\hline \multicolumn{5}{|l|}{ Age } \\
\hline$<50$ & 45 & 14 & 16.09 & \multirow[t]{2}{*}{0.503} \\
\hline$\geq 50$ & 42 & 14 & 16.09 & \\
\hline \multicolumn{5}{|l|}{ Wound site } \\
\hline Extremities & 74 & 24 & 85.72 & \multirow[t]{3}{*}{0.850} \\
\hline Face/nose/mouth & 1 & 4 & 14.28 & \\
\hline Trunk & 2 & 0 & 0.00 & \\
\hline \multicolumn{5}{|l|}{ Vaccine after injury } \\
\hline Yes & 21 & 9 & 42.86 & \multirow[t]{2}{*}{0.286} \\
\hline No & 66 & 19 & 28.79 & \\
\hline \multicolumn{5}{|l|}{ Incubation period* } \\
\hline$<7$ days & 32 & 15 & 46.88 & \multirow[t]{2}{*}{0.033} \\
\hline >7days & 55 & 13 & 23.64 & \\
\hline \multicolumn{5}{|l|}{ Period of onset* } \\
\hline$<48$ hours & 59 & 21 & 35.59 & \multirow[t]{2}{*}{0.28} \\
\hline$>48$ hours & 28 & 7 & 25.00 & \\
\hline \multicolumn{5}{|l|}{ Fever } \\
\hline yes & 27 & 14 & 51.85 & \multirow[t]{2}{*}{0.013} \\
\hline no & 60 & 14 & 23.33 & \\
\hline \multicolumn{5}{|l|}{ Co morbidity } \\
\hline stress ulcer & 13 & 6 & 46.15 & \multirow[t]{7}{*}{0.002} \\
\hline respiratory distress & 2 & 0 & 0.00 & \\
\hline respiratory failure & 9 & 9 & 100.00 & \\
\hline autonomic storm & 15 & 12 & 80.00 & \\
\hline cardiac arrest & 2 & 2 & 100.00 & \\
\hline aspiration pneumonia & 2 & 2 & 100.00 & \\
\hline none & 44 & 0 & 0.00 & \\
\hline \multicolumn{5}{|l|}{ Grading } \\
\hline 2 & 12 & 2 & 16.67 & \multirow[t]{4}{*}{0.031} \\
\hline 3 & 45 & 11 & 24.44 & \\
\hline 4 & 23 & 10 & 43.48 & \\
\hline 5 & 7 & 5 & 71.43 & \\
\hline \multicolumn{5}{|c|}{$\begin{array}{l}\text { Use of mechanical ventilation } \\
\text { or tracheostomy }\end{array}$} \\
\hline yes & 61 & 22 & 36.07 & \multirow[t]{2}{*}{0.318} \\
\hline no & 26 & 6 & 23.08 & \\
\hline
\end{tabular}

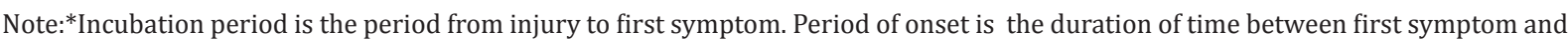
occurrence of spasm

Althea Medical Journal. 2015;2(2) 
The second highest was between 50-64 years which was $29(32.18 \%)$. The lowest was presented between 20-34 years which was 10 (11.49\%), followed by the age > 65 years which was $14(16.09 \%)$. The most common wound site was extremities which were presented in $74(85.07 \%)$ patients, followed by face/nose/ mouth $11(12.65 \%)$. The least common wound site was trunk which was two $(2.30 \%)$. The absence of post-injury vaccination was shown as majority $66(75.86 \%)$. Only $21(24.24 \%)$ were vaccinated after injury. Based on the grading, most patient were presented with tetanus grade 3 which is 45 (51.73\%) of them. It was followed by tetanus grade 4 which was $23(26.44 \%)$, grade 2 which was $12(13.79 \%)$ and grade 5 which was 7 (8.05\%) of them.

The mortality rate among cases with an incubation period of $<7$ days was higher (46.88\%). Fourteen (51.85\%) patients died with the presence of fever. In several comorbidities of autonomic storm have shown the highest mortality rate with $12(80 \%)$ patients. Mortality was high in patients for the period of onset $<48$ hours which was 21 (35.59\%). Patients who had been through mechanical ventilation and tracheostomy and died were $22(36.07 \%)$.

Table 2 showed the relationship between mortality and prognostic factors for tetanus. Higher mortality rate was significantly associated with incubation period $<7$ days, presence of fever, co-morbidity of autonomic storm and more sever disease according to Patel and Joag grading. There were no significant association between age and sex, wound site, vaccine after injury, period of onset, and use of mechanical ventilation.

\section{Discussion}

Even though with the invention of tetanus toxoid, tetanus still cannot be eradicated in developing countries. ${ }^{7,8}$ The mortality rate is considered high at $32.18 \%$. Mortality was also proven high in a previous study by Polhaupessy. ${ }^{9}$ A high rate of tetanus is shown in male than in female which is in line with the study by Patel and Mehtra ${ }^{10}$, also in the study by Polhaupessy. ${ }^{9}$ This is due to higher exposure rate in dirty environment at work in male than in female. ${ }^{9,11}$ Besides, female are immunized before pregnancy. ${ }^{1,12}$ In this study, gender was not shown significant to mortality rate. It is only common that tetanus occurres more in male than in female.

The highest mortality rate of tetanus was shown in the age range between 35-49 years, $39.08 \%$. This may be due to the patients' activities at this age. Therefore, they are more prone to risk of injury which can be related to their work or other causes.13 In previous studies it was shown that there were associations of older age with mortality due to the poor adherence to immunization schedule. ${ }^{6}$ The study by Udwadia ${ }^{11}$ has stated that the response towards tetanus immunization declines at age more than 50 years old.

Vaccination after injury is important for preventing tetanus but in this study, the number of patients who were absence of postinjury vaccination is high (75.86\%).6 It is also proven in the study by Widjaya. ${ }^{13}$ Post-injury vaccinations are important in tetanus patients to prevent complications.

The factor wound site showed the highest number of patients was extremities with mortality rate $85.72 \%$. The results differences could be because the patients in this study who were from allow socioeconomic background and also had low education were not aware about the safety at work. Therefore, they were more likely to injure their extremities. Usually tetanus is more severe if the site of wound is at the face or cranial than at extremities.

The factor incubation period $<7$ days (46.88 \%; p 0.033) had a higher mortality compared to $>7$ days. This result was in line with the previous studies by Saltoglu et al..$^{5,6,10}$ The shorter the incubation period, the higher the risk of getting an infection showed that the toxin production by $\mathrm{C}$. tetani had not neutralized. This factor was proven to be significant.

The patients who were presented with fever were higher than those without fever. It is significantly related to mortality in tetanus as the previous studies also showed presence of fever is related to mortality. 6 In this study, fever was present because of the presence of microbiological organisms. Therefore, until the C. tetani was not eradicated the fever would not subside.

The co morbidity of autonomic storm had a $\mathrm{p}$ value of 0.002 . This showed it was significantly related to mortality. The study by Polhaupessy has proven that autonomic storm is significant to mortality with $\mathrm{p}<0.001{ }^{9}$ For severity of the disease by grade which is tetanus grade $V$ shows as the highest mortality which is in line with the previous studies, the severer the disease the higher the mortality rate. ${ }^{6,10,14}$ Grade $V$ is the severest stage where there is presence of the five criteria as stated earlier. Therefore, this shows the patient is 
in critical condition that will possibly lead them to further complications. This is proven significant to the mortality in tetanus.

The deaths from tetanus can be related to the prognostic factors. The factors which were significantly related to mortality were incubation period, presence of fever $(\mathrm{p}<0.05)$, co morbidity $(\mathrm{p}<0.01)$ and severity by grade $(\mathrm{p}<0.05)$. Hopefully, this study could provide guidance for the physician to put much more effort on the prevention aspect of tetanus to reduce the mortality rate.

The limitation to this study was management as a factor was not identified. A multivariate logistic analysis could be performed to identify the most significant factors affecting the mortality simultaneously.

In conclusion, the mortality rate of adult tetanus was high $(32.18 \%)$. The factors that were significantly affecting the mortality were incubation period less than 7 days, presence of fever, co morbid of autonomic storm and severity based by grade.

\section{References}

1. WHO. Immunization, vaccines and biological: tetanus. 2007 [Cited 2012 April 20]. Available from: http://www.who.int/ immunization/topics/tetanus/en/.

2. Gunawan D. Tetanus in adults in Bandung, Indonesia. Neurol J Southeast Asia.1996;1:43-6.

3. WHO. Immunization, vaccines and biological: immunization surveillance, assessment and monitoring. 2011 [Cited 2012 April 20]. Available from: http:// www.who.int/immunization/monitoring surveillance/en/.
4. Russell RWR, Wiles CM, editors. Integrated clinical sciences series: Neurology. London: Butterworth-Heinemann Medical Books Ltd; 1985.

5. Ramachandra L, Shobha KL, Kannan PA. A retrospective clinical study of factors affecting tetanus. The Internet Journal of Microbiology. 2008;7(1)

6. Saltoglu N, Tasova Y, Midikli D, Burgut R, Dündar IH. Prognostic factors affecting deaths from adult tetanus. Clin Microbiol Infect. 2004;10(3):229-33.

7. WHO. Tetanus. 2012 [downloaded in 20 April 2012]. Available at: http://www. who.int/topics/tetanus/en/

8. Polhaupessy D. Pola dan pengaruh disotonomia terhadap prognosis pasien tetanus [dissertation]. Bandung: Universitas Padjajaran; 1999.

9. Patel JC, Mehta BC. Tetanus: study of 8,697 cases. Indian J Med Sci. 1999;53(9):393401.

10. Udwadia F. Tetanus [dissertation]. Delhi: Bombay Oxford University Press; 1994.

11. Centers for Disease Control and Prevention. Vaccines and immunizations: vaccines \& preventable diseases. 2009 [Cited 2012 April 20]. Available from: http://www.cdc. gov/vaccines/vpd-vac/tetanus/default. htm.

12. Widjaya RK. Perbandingan Penggunaan HTIG 500 IU dengan ATS EQUINE10000 IU terhadap Keluaran Pasien Tetanus [dissertation]. Bandung: Universitas Padjadjaran; 2007.

13. Owolabi LF, Habib AG, Nagoda M. Predictors of mortality among adult tetanus patients in Northwestern Nigeria. Neurology Asia. 2011;16(3):199-203. 